\title{
Expert System of Electric Pump Well Condition Diagnosis
}

\author{
Jia Zhang, 2, a \\ ${ }^{1}$ Yangtze University College of Technology \& Engineering, Jingzhou 434020, China \\ ${ }^{2}$ State Key Laboratory of Software Engineering, Wuhan University, Wuhan 430072, China \\ azhangjia027@163.com
}

Keywords: Expert system; Electric pump well; Diagnosis; Fault tree; Rete algorithm

\begin{abstract}
In order to realize the intelligent diagnosis of the electric pump and the motor working condition during the operation period of the electric pump well, an expert system based on fault tree and rete algorithm is realized. Through the analysis on the fault characteristics of the electric pump well, established the fault tree and knowledge base that support electric pump and motor working condition diagnosis, then combined with the rete algorithm based on production rule, which is used to obtain the diagnosis result of working condition, and gives the corresponding optimization suggestion. At last, based on the basic data and motor test data of electric pump well, the working conditions of 57 wells were diagnosed. The result shows that the diagnosis results are consistent with the practice, which improves the efficiency and accuracy of the working condition's fault location.
\end{abstract}

\section{Introduction}

As kind of mechanical oil extraction equipment, electric submersible pump has the characteristics of large displacement, long working life of underground and convenient management.These years has been widely used in the oil field. But the system efficiency of electric pump well is not high due to the reasons of pump selection, lack of fluid, tubing leakage and plug [1]. Therefore, it is necessary to diagnose the condition of the electric pump well, so as to take the corresponding measures to eliminate the faults, and make the system to be superior. At present, there are lots of electric pump well condition diagnosis method [2], in addition to the traditional current card diagnosis method, hold pressure diagnosis method, system efficiency control chart analysis method, also includes the diagnosis method based on the fuzzy mathematics theory and BP neural network $[3,4]$. Niu $x$ et al proposed a fault diagnosis method based on BP neural network that is applied to the fault diagnosis of high voltage circuit breaker [5]. With the development of artificial intelligence technology, the fault diagnosis method based on expert system has been applied to the relevant industries. An expert system is designed to help the doctor or expert in making the appropriate diagnosis of the patient [6]. An expert system based on fault tree and neural network is used in control box fault diagnose of digita control system [7].

Fault tree (Tree Analysis Fault, FTA) is an important analysis method for fault diagnosis [7]. On the other hand, expert system reasoning based on rule engine, and rete algorithm is an efficient pattern matching algorithm for the production rule system [8]. So it is necessary to in-depth analysis of the FTA and rete algorithm, to achieve the precise diagnosis of electric pump wells.

\section{The Establishment of Fault Tree and Knowledge Base}

Fault Analysis of Electric Pump Well: The working condition diagnosis of electric pump well is divided into the diagnosis of electric pump and the motor. It is necessary to analysis the main features of the electric pump well system and classify the working conditions. The following is the part of the working conditions:

- Motor underload, the main features: running current is less than $85 \%$ of the rated current.

- Motor overload, the main features: running current greater than $115 \%$ of the rated current, the motor output power is greater than the rated power. 
- Motor or cable insulation decreased, the main features: current unbalance degree is greater than $5 \%$.

- Pump or tubing leakage, the main features: pump efficiency is low. The dynamic liquid level is high, and the running current is less than the rated current.

- The liquid supply is insufficient, the main features: running current is less than the rated current, the pump inlet pressure is small, the output is small, and the dynamic liquid level is low.

The Establishment of Fault Tree: Fault tree analysis (FTA) is an important analysis method for fault diagnosis. Various faults are described in an inverted tree structure according to the internal cause and relationship. In fault analysis, the direct causes and indirect causes of the fault can be found out from a point of failure. This analysis method can accurately locate the fault, and it is easy to understand.

The complexity and variety of the fault condition have put forward higher request to the accurate diagnosis of the electric pump well condition, but there is a certain causal relation between these faults, which can be described by the fault tree. The most important two elements in the fault tree are as follows:

- Event: Mainly divided into top event, basic event and intermediate event. The top event is the starting point for the current fault diagnosis, that is the fault to investigate; Basic event is the ultimate causes of other events, and there is no need to further explore the reason for the occurrence; Intermediate event is the event that between top event and basic event.

- Logic gate symbol: Used to describe the logical relations between events, including AND, OR and NOT gates.

In the diagnosis of motor working condition, as the motor efficiency is an important indicator to measure the motor condition, three levels of the motor working condition can be obtained by this index: good (efficiency is greater than 70\%), the general (efficiency is between $40 \%$ to $70 \%$ ) and poor (efficiency is less than 40\%). Therefore, these three levels can be used as the top event in the fault tree, the following is the "motor condition is poor" as an example, the establishment of the fault tree, as shown in Fig. 1.

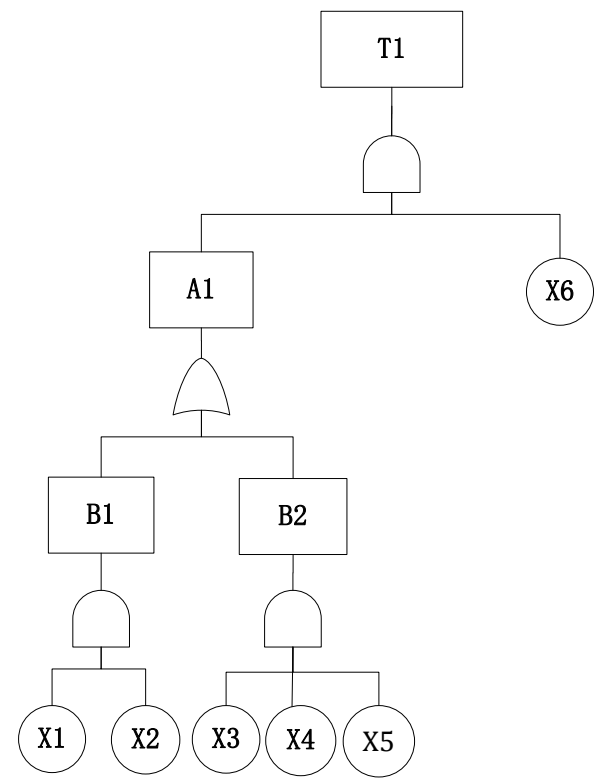

(a)
TI:Poor motor condition A1:Current and voltage abnormity B1:Lack of phase of power supply system B2:0perating current increases $\mathrm{X} 1: \mathrm{I} 0>5 \%$ $\mathrm{X} 2: \mathrm{V} 0>10 \%$ $\mathrm{X} 3: \mathrm{I} 0>5 \%$ $\mathrm{X} 4: \mathrm{V} 0<115 \% \mathrm{Ie}$ $\mathrm{X} 5: \bar{V}<90 \% \mathrm{Ve}$ $\mathrm{X} 6: \mathrm{N}_{0}>20 \%$

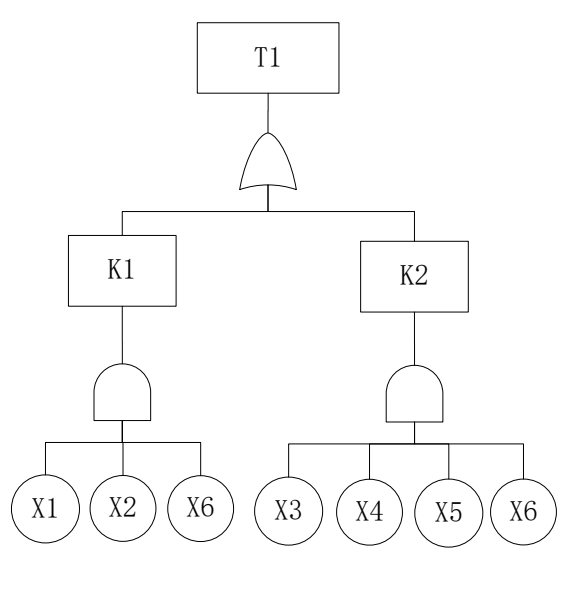

(b)

Figure 1. Example of fault tree diagnosis for motor condition of electric pump well

The Establishment of Knowledge Base: As shown in Fig. 1(a), $\mathrm{T}_{1}$ is top event; A1, B1 and B2 are intermediate events; X1 to X6 are the basic events. According to the fault tree, the combination of the basic events leading to the occurrence of the top event can be obtained based on qualitative 
analysis. The minimal cut set of the fault tree is one of the most important methods used in the qualitative analysis, which is based on the Boolean algebra rules.

The slove process of the minimum cut set for the top event $\mathrm{T} 1$ is as follows:

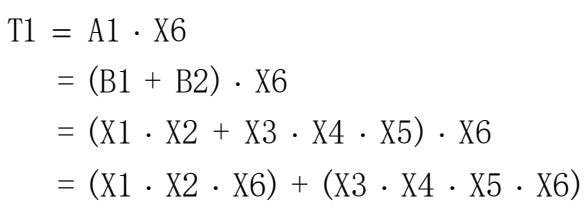

As shown in Eq.1, fault tree minimal cut set is converted to: $K_{1}=\left(X_{1} X_{2} X_{6}\right), K_{2}=\left(X_{3} X_{4} X_{5}\right.$ $\mathrm{X}_{6}$ ). Once the minimum cut set is obtained, which means that when a set of basic events occur, it may cause the occurrence of the top event,so the fault tree is simplified as shown in Fig. 2 according to the minimum cut set.

The knowledge in expert system is used to simulate the way of thinking of experts, and the knowledge base is used to store knowledge. In the field of artificial intelligence, knowledge expression has a variety of ways, such as production, semantic web, etc. Production rule is widely used in expert system, its basic form is IF... THEN..., if a particular condition is met, a result is produced. Similar to programming language syntax, IF can be followed by a number of conditions that connect with the logical operator AND, OR, NOT.

Each of the minimum cut sets corresponds to a failure pattern of the top event, and for the knowledge base, every minimum cut set corresponds to knowledge. When building a knowledge base based on the production rule, each cut set is converted into a rule. Such as $\mathrm{R}_{1}$ that converted from $\mathrm{K}_{1:}$ IF $\mathrm{X}_{1}$ AND X $\mathrm{A}_{2}$ AND $\mathrm{X}_{6} \mathrm{THEN} \mathrm{T}_{1}$.

\section{Rule Reasoning Based on Rete Algorithm}

The Rete algorithm is a pattern matching algorithm based on production rule, which is proposed by Dr. forgy - Carnegie Mellon University in the United States. Rete algorithm consists of two parts, the rule compilation that according to that rule set corresponding reasoning network; another part is runtime execution that sends the fact data to the reasoning network for rule matching $[9,10]$.

Rete network can be further divided into alpha network and beta network, alpha network is used to describe the rules of the system, and beta network used to describe the matching results. In Rete reasoning network, the following types of nodes are mainly included:

- RootNode: the root node, entrance of the reasoning network;

- ObjectTypeNodes: type node, node type filter for pattern matching;

- AlphaNode: stored in the alpha network, describe the pattern of the rule;

- JoinNode: stored in the beta network, describe the intermediate result for the pattern matching;

- TerminalNode: the end node, which matching the corresponding rules and waiting for the next step.

Rule $\mathrm{R}_{1}$ : "IF $\mathrm{X}_{1}$ AND $\mathrm{X}_{2}$ AND $\mathrm{X}_{6}$ THEN $\mathrm{T}_{1}$ " as an example $\left(\mathrm{X}_{1}, \mathrm{X}_{2}, \mathrm{X}_{6}\right.$ is the three patterns of the rule), the establishment of the local rete reasoning network as shown in Fig. 2. 


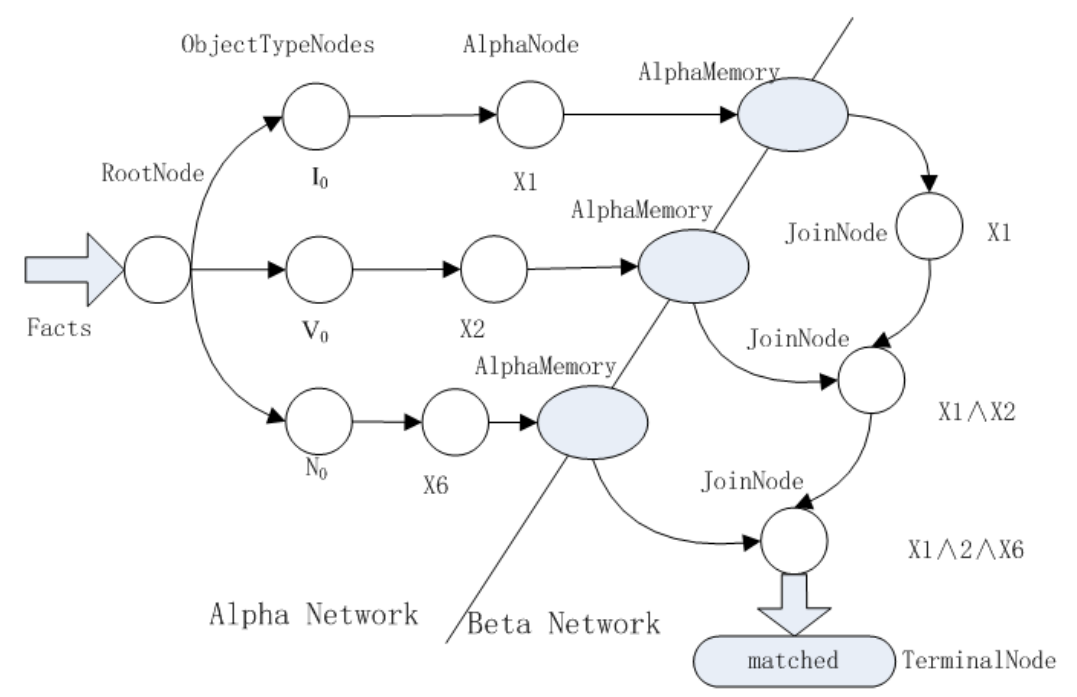

Figure 2. Rete reasoning network

The specific process is as follows:

Step 1: Create a root node (root node) as the entrance of the inference network.

Step 2: Get the pattern $\mathrm{i}(\mathrm{i}=\{1,2,3\})$ from the rule 1 (the pattern is the smallest atomic condition, so the relationship between rule and pattern is $1: n)$.

Step 3: check the parameter type in the pattern $i$, if it is a new type, add a TypeNode.

Step 4: check the pattern i corresponding to the AlphaNode exists, if there is, record the location of the node; if not, the pattern $\mathrm{i}$ is added to the network as a AlphaNode. At the same time, according to the AlphaNode to refresh the Alpah memory table.

Step 5: repeat the step2 - 4 until all patterns are processed.

Step 6: construction the JoinNode of Beta network. JoinNode has two input nodes, one node is JoinNode (i-1), and the other node is AlphaNode (i).

Step 7: repeat step 6 until all Beta nodes are processed.

Step 8: the Then part of the rule is encapsulated as the last node as JoinNode (n).

\section{Summary}

The condition of electric pump well is complicated, base the fault tree and the minimal cut set method can simplify the complex working condition and get the basic event of the fault. Rete algorithm is used to reasoning based production rule, and the performance of rule reasoning is improved by using cache of the intermediate result. The results show that the system can well support the diagnosis of electric pump well, save energy consumption for oil field, and bring some economic benefits.

\section{Acknowledgments}

This work was supported by Project of Hubei Province Education Science Planning 2015 (2015GB165).

\section{References}

[1] F.Y.Tao, G.F.Liu and X.G.LI. Research on Fault Feature Extraction of the Submersible Pump Units. Noise \& Vibration Control, 2012,32(2):140-142.

[2] C.Zheng, X.Hao and X.Huang. Application of holding pressure diagnosis techniques in management of submersible electric pump well in Bohai Q Oilfiel. Oil Drilling \& Production Technology, 2012.34(1):59-62. 
[3] J.Q.Xue, M.H.Li and G.D.Zhang, et al. Fault diagnosis technique of screw pump wells based on BP neural network. Journal of Xian Shiyou University, 2013, 28(3):74-77.

[4] J.Zhu, Y.Gao and X.Ye. Study of screw pump well fault diagnosis based on BP neural network. China Petroleum Machinery, 2008, 36(1):42-44.

[5] X.Niu, X.Zhao. The Study of Fault Diagnosis the High-Voltage Circuit Breaker Based on Neural Network and Expert System. Procedia Engineering, 2012, 29:3286-3291.

[6] Singla J. The Diagnosis of Some Lung Diseases in a Prolog Expert System. International Journal of Computer Applications, 2013, 78(15):37-40.

[7] Wang Y, Li Q, Chang M, et al. Research on Fault Diagnosis Expert System Based on the Neural Network and the Fault Tree Technology. Procedia Engineering, 2012, 31(16):1206-1210.

[8] Forgy C L. Rete: A fast algorithm for the many pattern/many object pattern match problem[J]. Artificial Intelligence, 1982, 19(82):17-37.

[9] Liu D, Gu T, Xue J P. Rule Engine based on improvement Rete algorithm[C]// Apperceiving Computing and Intelligence Analysis (ICACIA), 2010 International Conference on. IEEE, 2010:346 - 349.

[10] Kawakami T, Yoshihisa T and Yanagisawa Y, et al. A Rule Processing Scheme Using the Rete Algorithm in Grid Topology Networks[C]// 2015 IEEE 29th International Conference on Advanced Information Networking and Applications (AINA). IEEE Computer Society, 2015:674-679. 